\title{
Representação social do consumo de álcool em idosos de uma população quilombola
}

\section{Bruna Ramos Neves}

(D) https://orcid.org/

Thainara Araujo Franklin ${ }^{1}$

(D) https://orcid.org/0000-0003-2065-5090

Tito Lívio Ribeiro Gomes do Nascimento ${ }^{1}$

(D) https://orcid.org/0000-0001-5324-711X

Soraya Mendes Rodrigues Adorno ${ }^{1}$

(iD https://orcid.org/

Alba Benemérita Alves Vilela

(DD https://orcid.org/0000-0002-1187-0437

Universidade Estadual do Sudoeste da Bahia, Jequié, BA, Brasil.
Objetivo: compreender as palavras evocadas por idosos de uma população quilombola acerca do consumo de álcool. Método: trata-se de uma pesquisa de abordagem mista (quanti-qualitativa). Utilizou-se a Técnica de Evocação Livre de Palavras com o termo indutor "Consumo de bebidas alcoólicas". A análise foi realizada através do software EVOC 2005. Utilizou-se um formulário com questões sociodemográficas, com análise no software SPSS, versão 21. Resultados: foram entrevistados 60 idosos, a maioria do sexo feminino e com baixa escolaridade. Conclusão: as representações sociais acerca do consumo de bebidas alcoólicas se mostraram negativas, o que emerge significações atreladas ao alto consumo de álcool e à problematização advinda com este.

Descritores: Idoso; Quilombo; Representações Sociais; Alcoólicos.

\section{Como citar este artigo}

Neves BR, Franklin TA, Nascimento TLRG, Adorno SMR, Vilela ABA. Social representation of alcohol consumption among elders of a quilombola population. SMAD, Rev Eletrônica Saúde Mental Álcool Drog. 2019;15(4):1-8. doi: https://dx.doi.org/10.11606/issn.1806-6976.smad.2019.153605 


\title{
Social representation of alcohol consumption among elders of a quilombola population
}

\begin{abstract}
Objective: understand the words evoked by elders of a quilombola population about alcohol consumption. Method: this is a mixed-approach (quantitative and qualitative) research. The Free-Speech Evoking Technique was used with the inductive term "alcohol consumption". The analysis was performed using EVOC 2005 software. A form with sociodemographic questions was used, and the statistical analysis was performed with SPSS software, version 21. Results: participants interviewed were 60 elderly, mostly were female and with low schooling. Conclusion: the social representations on the alcohol consumption were negative, which shows meanings linked to the high consumption of alcohol and to the problems deriving from this consumption.
\end{abstract}

Descriptors: Elderly; Quilombo; Social Representations; Alcoholics.

\section{La representación social del alcohol en una población de edad avanzada quilombo}

Objectivo: comprender las palabras evocadas por ancianos de una población quilombola acerca del consumo de alcohol. Metodo: se trata de una investigación de enfoque mixto (cuantitativa). Se utilizó la Técnica de Evocación Libre de Palabras con el término inductor "Consumo de bebidas alcohólicas" el análisis fue realizado a través del software EVOC 2005. Se utilizó un formulario con cuestiones sociodemográficas, con análisis en el software SPSS versión 21. Resultados: fueron entrevistados 60 ancianos, mayoría del sexo femenino y con baja escolaridad. Conclusión: las representaciones sociales, acerca del consumo de bebidas alcohólicas se mostraron negativas, lo que emerge significaciones atadas al alto consumo de alcohol y la problematización que viene con este.

Descriptores: Ancianos; Quilombo; Representaciones Sociales; Alcohólicos. 


\section{Introdução}

O envelhecimento populacional está ocorrendo de forma rápida e distinta ao longo do tempo, trazendo desafios, principalmente para os países em desenvolvimento, levando a importantes responsabilidades sociais e econômicas. A prevalência de doenças crônico-degenerativas, nessa fase da vida, reforça a necessidade de ações eficazes no sentido de promover melhor qualidade de vida aos idosos ${ }^{(1)}$.

Estima-se que, em 2050, o número de pessoas com mais de 60 anos, no mundo, passará de 12,3\% para $21,5 \%$. No Brasil, o ritmo deve ser ainda mais acelerado, no entanto, nos próximos 35 anos, os idosos devem passar de 12,5\% (23 milhões) para 30\% (64 milhões) da população do país. Assim, passaríamos a ser, então, uma nação envelhecida (classificação dada aos países com mais de $14 \%$ da população constituída por pessoas da terceira idade) $)^{(2)}$.

Embora o envelhecimento seja um processo natural no qual o organismo passa por diversas alterações anatômicas e funcionais, com repercussões nas condições da saúde do idoso, associadas às alterações decorrentes do envelhecimento, mudanças como a aposentadoria, perda de amigos, solidão e isolamento social tornam os idosos vulneráveis e mais propensos à intensificação de hábitos menos saudáveis, como o consumo abusivo do álcool(1).

Pode-se dizer que o alcoolismo é uma doença crônica, progressiva e potencialmente fatal, que pode ser caracterizada por sintomas como uso abusivo do álcool e uma constante preocupação com a ingestão de bebida, predominando o seu consumo, apesar das consequências adversas ${ }^{(3)}$. Os prejuízos provocados pelo abuso do álcool ocasionam danos físicos e mentais, caracterizando-se como um problema de saúde pública, além disso contribuem para danos sociais, causando muitas vezes desagregação familiar.

A identificação epidemiológica dos segmentos sociodemográficos mais suscetíveis ao consumo e à dependência alcoólica é de suma importância, uma vez que permite avaliar as particularidades referentes ao consumo excessivo, por exemplo, as diferenciações entre os padrões de consumo e a cor da pele. De acordo com o Conselho Nacional antidrogas, a prevalência do abuso do álcool é mais presente na população negra(4).

A partir das limitações desse indicativo, a cor da pele pode captar as desigualdades em saúde às quais grupos sociais como os quilombolas estão expostos. Esses grupos sociais se distinguem dos demais pela identidade étnica através de critérios de autoatribuição e trajetória histórica própria(5).

Estudos apontam que a população negra do Brasil ocupa posições menos qualificadas e de pior remuneração no mercado de trabalho. A população quilombola reside em áreas com ausência ou baixa disponibilidade de serviços de infraestrutura básica; sofre maiores restrições no acesso a serviços de saúde e, estes, quando disponibilizados, são de pior qualidade e menor resolutividade(6).

Devido às desigualdades vistas no Brasil, principalmente nas comunidades quilombolas, foi criada a Política Nacional de Saúde Integral da População Negra (PNSIPN) que tem por objetivos garantir e ampliar o acesso à população negra do campo e da floresta, em particular as comunidades quilombolas, às ações e aos serviços de saúde, como também identificar as necessidades de saúde destas, utilizando como critério de planejamento e definição de $\operatorname{prioridades}^{(7)}$.

Nesse contexto surge a proposta deste estudo, o qual pretende compreender as palavras evocadas por idosos de uma população quilombola, partindo do termo indutor consumo de bebidas alcoólicas, uma vez que a Teoria das Representações Sociais - TRS é uma teoria capaz de apreender aspectos tão sutis da racionalidade humana e das relações sociais, levandonos a compreender os fenômenos que acontecem em nosso meio.

Nessa perspectiva, pode-se afirmar que as representações sociais- RS não significam reproduzir, duplicar, mas, sim, reconstruir, a partir de seus valores, crenças e normas próprias. Assim, propõe-se estudar a temática partindo da seguinte questão norteadora: O que pensam os idosos quilombolas a respeito do consumo de bebidas alcóolicas?

\section{Método}

O presente estudo trata-se de uma pesquisa de abordagem mista devido à presença de análises quantitativas e qualitativas no presente estudo, do tipo inquérito domiciliar de base populacional, buscando um aporte teórico na Teoria das Representações de Moscovici(8). Apresenta como sujeitos os idosos que residem na Comunidade Remanescente de Quilombos, na área de abrangência de uma Unidade Básica de Saúde da Família (UBSF), em um município de médio porte, localizado no sudoeste da Bahia.

As visitas foram realizadas em duas microáreas, nos meses de julho e agosto de 2016. A área de abrangência da UBSF possui 113 idosos residentes. Após os critérios de inclusão e exclusão, ficou definida uma amostra potencial de 83 idosos, dos quais foram entrevistados 60 idosos, 23 idosos não foram encontrados em casa, após três tentativas.

Como critérios de inclusão estabelecidos para a constituição da amostra deste estudo, os entrevistados deveriam ser idosos, com idade igual ou superior a 60 (sessenta) anos, cadastrados na USF em estudo. E como critérios adotados para a supressão da participação 
do idoso nesta investigação, estabeleceu-se em não responder na íntegra aos questionários ou se recusar a participar da pesquisa.

A presente pesquisa foi aprovada pelo Comitê de Ética em Pesquisa da Universidade Estadual do Sudoeste da Bahia, perante o número de identificação 047/2009, de acordo com os preceitos da Resolução 196/96 do Conselho Nacional de Saúde. Respeitando-se os sujeitos da pesquisa quanto à participação no estudo, como disponibilização do Termo de Consentimento Livre e Esclarecido antes da aplicação.

Os elementos qualitativos foram advindos através de uma técnica ou teste de evocação livre de palavras ${ }^{(9)}$, tendo como termo indutor consumo de bebidas alcoólicas, sendo orientada a produção de no máximo cinco palavras que viessem à mente a partir do termo proposto. Após a evocação, solicitou-se que os sujeitos realocassem as palavras por ordem de importância. Criou-se um dicionário a partir das palavras evocadas, em que para palavras com aproximação semântica foi dado o mesmo sentido (ex.: Prejudicial e Prejuízo, substituiu-se Prejudicial por Prejuízo).

O uso da técnica de associação livre de palavras no campo das representações sociais se difere pela aplicação de análise estrutural em investigação científica, assim é construído para cada termo indutor um corpus de palavras e variáveis quantitativas: frequência intermediária obtida pela média, sabendo-se que a frequência das evocações não se distribui uniformemente; frequência mínima, a qual é determinada pelo ponto de corte em que excluem uma parte do banco de dados sem significado e ordem média de evocação (OME) que é calculada pela média ponderada pela razão entre a posição da palavra evocada e sua frequência(7).

A partir do cruzamento do corpus ordenado por importância e as variáveis que já foram citadas, o material será calculado e cada corpus, ao final, gerará os quadros de quatro casas, incluindo os conteúdos e identificando palavras em zonas de núcleo central (quadrante superior esquerdo) com uma maior frequência e importância; zonas periféricas (quadrante ao lado esquerdo) com uma frequência maior e menor e menos importantes; e zonas de elementos de contrastes (quadrante inferior direito) com uma frequência menor e mais importante para aqueles que evocam ${ }^{(10-11)}$.

As informações quantitativas foram provenientes de um formulário com questões sociodemográficas e de saúde de autoria dos autores do estudo. Para a análise descritiva dos dados, utilizou-se o software SPSS, versão 21 , e as análises do corpus das palavras evocadas foram apoiadas nos elementos teóricos da abordagem estrutural das representações sociais com a padronização das palavras por similaridade e uniformização dos termos por gênero e número. Os cálculos foram feitos com o auxílio do software Ensemble de Programmes Permettantl Analysedes Evocations (EVOC), versão 2005, sendo apresentados os resultados em um quadro de quatro casas.

\section{Resultados}

Conforme a Tabela 1, a pesquisa evidenciou que a média de idade dos 60 idosos quilombolas entrevistados é maior na faixa etária de 60 -69 anos de idade, correspondendo a $50 \%$, sendo $53,3 \%$ do sexo feminino.

Tabela 1 - Características sociodemográficas e econômicas de pessoas idosas quilombolas em Jequié, BA, 2016

\begin{tabular}{|c|c|c|}
\hline Variável & $\mathrm{n}=60$ & $\%$ \\
\hline \multicolumn{3}{|l|}{ Sexo } \\
\hline Masculino & 28 & 46,7 \\
\hline Feminino & 32 & 53,3 \\
\hline \multicolumn{3}{|l|}{ Faixa Etária } \\
\hline $60-69$ & 30 & 50 \\
\hline $70-78$ & 22 & 36,7 \\
\hline $80-88$ & 6 & 10 \\
\hline $92-94$ & 2 & 3,3 \\
\hline \multicolumn{3}{|l|}{ Escolaridade } \\
\hline Nenhuma & 33 & 55 \\
\hline Fundamental I Incompleto & 22 & 36,7 \\
\hline Fundamental I Completo & 5 & 8,3 \\
\hline \multicolumn{3}{|l|}{ Estado Conjugal } \\
\hline Solteiro(a) & 11 & 18,3 \\
\hline Casado(a)/Morando junto(a) & 30 & 50,0 \\
\hline Viúvo(a) & 12 & 20,0 \\
\hline Divorciado(a)/Separado(a) & 7 & 11,7 \\
\hline \multicolumn{3}{|l|}{ Renda do Idoso } \\
\hline Sem renda & 6 & 10 \\
\hline$=1 \mathrm{SM}^{*}$ & 39 & 65 \\
\hline$<1 \mathrm{SM}^{*}$ & 9 & 15 \\
\hline$>1 \mathrm{SM}^{*}$ & 6 & 10 \\
\hline \multicolumn{3}{|c|}{ O que ganha para as necessidades básicas } \\
\hline Dá e sobra & 4 & 6,7 \\
\hline Dá na conta certa & 35 & 58,3 \\
\hline Sempre falta um pouco & 13 & 21,7 \\
\hline Sempre falta muito & 8 & 13,3 \\
\hline \multicolumn{3}{|c|}{ Situação econômica aos 50 anos de idade em relação à atual } \\
\hline Melhor & 37 & 61,7 \\
\hline A mesma & 7 & 11,7 \\
\hline Pior & 16 & 26,6 \\
\hline
\end{tabular}

*SM = salário mínimo R\$ 880,00, Brasil, 2016

Conforme observado na Tabela 1, a maioria dos idosos entrevistados não possuía nenhum tipo de escolaridade, correspondendo a 55\%. Para $61,7 \%$ dos entrevistados, a situação econômica atual foi classificada como melhor do que quando tinham 50 anos de idade e 58,3\% consideraram a renda atual suficiente para suprir as necessidades básicas da família, sendo na 
conta certa. Em relação ao estado conjugal, 50\% dos indivíduos são casados ou moram juntos.

$\mathrm{Na}$ análise das representações sociais houve um corpus produzido pelos idosos com a presença de 296 palavras perante o termo indutor "consumo de bebidas alcoólicas", sendo 65 destas diferentes. A média das ordens médias de evocação (rang) encontrada foi de 2,95, sendo ajustada para 3 ; já a frequência intermediaria, considerando que foram desprezadas evocações cuja frequência foi igual ou menor que 3, encontrou-se a frequência média igual a 10 . Sendo assim, pode-se através do programa EVOC 2005 construir o quadro de quatro casas conforme a Figura 1.
Na Figura 1, evidenciam-se as seguintes palavras evocadas: no quadro superior esquerdo, prejuízo, ruim, vício e violência, sendo estas os possíveis elementos centrais da representação; o quadro superior direito apresenta as seguintes palavras: agressividade, briga, doença, embriaguez e saúde, sendo estas os elementos de primeira periferia; no quadro inferior esquerdo: bar, cachaça, destruição, dinheiro, errado, problema, sentimento, tristeza, caracterizados como os elementos de segunda periferia das representações; e por fim, o quadro inferior direito, contendo as seguintes palavras: alegre, cerveja, chateação, deixar, descontrole, família, fumo, morte e sofrimento.

\begin{tabular}{|c|c|c|c|c|c|}
\hline \multicolumn{3}{|c|}{ Elementos centrais } & \multicolumn{3}{|c|}{ Elementos intermediários $-1^{\circ}$ periferia } \\
\hline \multicolumn{2}{|c|}{ Frequência $>=10$} & \multirow{2}{*}{$\begin{array}{c}\text { Rang }<3,0 \\
\text { Rang }\end{array}$} & \multicolumn{2}{|c|}{ Frequência $>=10$} & \multirow{2}{*}{$\begin{array}{c}\text { Rang }>3,0 \\
\text { Rang }\end{array}$} \\
\hline Evocações & Freq. & & Evocações & Freq. & \\
\hline Prejuizo & 26 & 2,500 & Agressividade & 11 & 3,727 \\
\hline Ruim & 21 & 1,857 & Briga & 17 & 3,706 \\
\hline Vício & 28 & 2,143 & Doença & 30 & 3,600 \\
\hline \multirow[t]{2}{*}{ Violência } & 11 & 2,364 & Embriaguez & 12 & 3,417 \\
\hline & & & Saúde & 10 & 3,600 \\
\hline \multicolumn{3}{|c|}{ Elementos de contraste } & \multicolumn{3}{|c|}{ Elementos periféricos $-2^{\circ}$ periferia } \\
\hline \multicolumn{2}{|c|}{ Frequência $<10$} & Rang $<3,0$ & \multicolumn{2}{|c|}{ Frequência $<10$} & Rang $>3,0$ \\
\hline Evocações & Freq. & Rang & Evocações & Freq. & Rang \\
\hline Bar & 4 & 2,500 & Alegre & 6 & 3,333 \\
\hline Cachaça & 6 & 1,833 & Cerveja & 7 & 3,857 \\
\hline Destruição & 6 & 2,500 & Chateação & 3 & 3,333 \\
\hline Dinheiro & 4 & 2,750 & Deixar & 5 & 3,200 \\
\hline Errado & 5 & 3,000 & Descontrole & 8 & 3,625 \\
\hline Problema & 3 & 2,667 & Família & 4 & 4,750 \\
\hline Sentimento & 3 & 2,667 & Fumo & 3 & 4,000 \\
\hline \multirow[t]{2}{*}{ Tristeza } & 9 & 3,000 & Morte & 7 & 3,571 \\
\hline & & & Sofrimento & 4 & 3,500 \\
\hline
\end{tabular}

Rang = 3,0; frequência mínima $=3$; frequência média $=10$

Figura 1 - Quadro de quatro casas ao termo indutor "Consumo de bebidas alcoólicas". Idosos Quilombolas, Jequié/Ba, Brasil, 2016

\section{Discussão}

Verificou-se que a maioria dos idosos entrevistados $(53,3 \%)$ foi do sexo feminino, corroborando com dados da Pesquisa Nacional por Amostra de Domicílio, divulgada pelo Instituto Brasileiro de Geografia e Estatística - IBGE em $2010^{(12)}$, que indicam que vivem no Brasil 103,5 milhões de mulheres, o equivalente a $51,4 \%$ da população.

Em relação à faixa etária, 60-69 anos de idade corresponde a $50 \%$ dos entrevistados, o que indica que esse resultado se relaciona ao padrão demográfico brasileiro atual. Dados semelhantes são descritos pelo IBGE $(2012)^{(13)}$ que apontam que essa mesma faixa etária $(42,4 \%)$ é de maior representatividade nesse grupo populacional, em decorrência de uma transição demográfica relativamente recente ${ }^{(14)}$.
Quanto à variável escolaridade, verificou-se um alto percentual de entrevistados que não possuem escolaridade (55\%). A percepção da falta da oportunidade de estudo se mostra pela presença de pessoas analfabetas e/ou analfabetas funcionais, identificadas no decorrer do estudo. Atualmente, no Brasil, mais de 14 milhões de pessoas não conseguem ler, nem escrever um bilhete simples.

Além dessas pessoas, consideradas analfabetas, há também aqueles classificados como analfabetos funcionais(15). Em geral, um analfabeto funcional lê e escreve frases simples, mas não é capaz de interpretar textos, nem de colocar ideias no papel. Assim, pode-se dizer que o analfabetismo reflete nas condições de desigualdade social em que vivemos: a maioria daqueles 
que não sabem ler, nem escrever é de moradores da zona rural, negros e pardos.

Verificou-se também que $73,3 \%$ dos idosos possuem uma renda de até um salário mínimo. A renda constitui um importante recurso em muitas das ocasiões, $58,3 \%$ dos idosos entrevistados relataram acerca da suficiência da renda familiar para o suprimento das necessidades básicas apenas "dá na medida certa". E embora os resultados tenham apresentado que a maioria dos idosos possui uma baixa renda familiar, $61,7 \%$ dos idosos avaliaram a sua atual situação econômica como "melhor que aos 50 anos de idade".

Em relação às $\mathrm{RS}$, para melhor compreensão da leitura dos resultados, cabe esclarecer que quanto menor for o rang de cada palavra, mais prontamente ela foi evocada, e quanto maior o rang, isso significa que foi evocada mais tardiamente. Observa-se nos resultados que o significado de "consumo de bebida alcóolica" para os idosos é fortemente marcado por elementos negativos, identificados pelas palavras em núcleo central, prejuízo, ruim, vício e violência.

A palavra prejuízo que tem a maior frequência e que foi mais prontamente evocada demonstra uma dimensão em que os idosos associam esse comportamento a riscos de complicações evidenciadas no seu passado ou sendo vivenciadas na atualidade, sendo estas dificuldades financeiras, problemas de saúde, dentre outros problemas que são comuns na população quilombola. O resultado reflete, também, o posicionamento dos sujeitos perante o problema, por meio de emoções e atitudes, como ruim, vício e violência.

O termo "ruim" poderá estar associado ao quadro de complicações que o álcool pode causar, principalmente ao longo dos anos, e pelo abuso excessivo, tornando-se um vício. Segundo a Organização Mundial de Saúde(16), o alcoolismo é considerado uma doença crônica, com aspectos socioeconômicos e comportamentais, ou seja, é uma substância fortemente ligada a mudanças de hábitos que podem resultar em histórias trágicas de violência(17). Sendo assim, a palavra violência sugere práticas sociais dispostas nesse grupo acerca da violência no seu cotidiano intra ou extrafamiliar.

Os elementos periféricos de uma representação social estabelecem uma ligação entre o núcleo central e a realidade concreta na qual são elaboradas e funcionam essas representações ${ }^{(18)}$. Pode-se observar que o resultado apresentado é compatível com essa afirmação, tendo em vista que os elementos do núcleo central expressam mais elementos subjetivos e, de igual forma, acontecem na periferia dessa representação, caracterizada por funções do sistema periférico como a concretização, regulação e a defesa( ${ }^{(9)}$.

A título de exemplo, a presença de elementos na zona de primeira periferia, como agressividade e briga, expressa meios de como surgiu a violência - elemento posto em núcleo central, assim como doença e saúde que se mostram interligadas ao processo de adoecer do indivíduo diante do alto consumo, mostrando-se ligadas aos termos postos em núcleo central - prejuízo e ruim.

No entanto, o alto consumo de álcool, caracterizado pela palavra embriaguez, mostra-se presente nas representações dos mesmos, sendo uma prática evidenciada na população negra. Diante disso, há uma concretização da palavra vício disposta em núcleo central. Pelo fato de o consumo de álcool desenvolver dependência e ter uma série de outros problemas de saúde, como a cirrose, ao longo do seu consumo, permite-se dizer que esse consumo é visto de um ponto negativo para o grupo da terceira idade.

O sistema periférico é responsável pela atualização e contextualização da representação. A periferia de uma representação social é considerada um "para-choque" entre a realidade e um núcleo central que não muda facilmente(19). Assim, através das palavras evocadas de representação da segunda periferia (chateação, descontrole, morte, sofrimento), foi possível relacionálas ao contexto central percebido, permitindo a ligação da dependência alcoólica, o que gera comorbidades aos indivíduos, o que se pode conduzir a uma fatalidade, a exemplo do óbito.

Outras palavras evocadas foram fumo e cerveja, em que a evocação de cerveja pode levar à compreensão de que as práticas sociais diante desse grupo são a ingestão desta, relacionando a alguns estudos nos quais a cerveja é a mais consumida( ${ }^{(20)}$, sendo também associada ao tabaco.

A palavra "alegre" pode estar relacionada a momentos considerados positivos, obtidos pela consumação da bebida alcoólica, assim como a palavra evocada "deixar" revela o desejo de muitos idosos em controlar ou deixar a ingestão, uma vez que os efeitos do alcoolismo sobre as relações humanas provocam prejuízos tanto ao alcoolista quanto a sua família, sendo esta outra palavra evocada.

As famílias experimentam prejuízos em diversas áreas. Isso porque são altos os níveis de conflito e tensão, com falta de clareza na organização familiar, que influem na confiança e segurança. Há diminuição da união entre seus membros, com isolamento interpessoal e prejuízo geral da comunicação, o que leva a dificuldades de convívio(21).

As representações através das palavras bar, cachaça, destruição, dinheiro, errado, problema, sentimento e tristeza constituem a zona de contraste. Esta comporta os elementos que vão caracterizar as variações das representações em função de subgrupos, sem, no entanto, modificar os elementos centrais e a própria representação, ou seja, denotam mudanças ou transição de uma representação social(22). Assim, esse 
conteúdo representacional é direcionado mais uma vez aos riscos de complicações da saúde e mortes, como também ao local onde se dá a consumação, relacionadas, assim, à ingestão frequente de bebidas alcoólicas.

\section{Conclusão}

O alcoolismo também pode atingir as pessoas idosas, gerando conflitos e situações de sofrimento no contexto familiar, social e pessoal. Por esse quadro, surge a necessidade de os profissionais tratarem não apenas a sintomatologia do idoso alcoolista, mas enxergá-lo como um todo, procurando conhecer a sua corresidência e assim proporcionar a recuperação do poder resiliente e fortalecer sua autoestima e o vínculo com os familiares. No entanto, os profissionais de saúde devem aprender a lidar com essa problemática cada vez mais presente nos lares brasileiros.

Nesse sentido, as RS dos idosos acerca do consumo de bebidas alcoólicas são ancoradas pelas vivências de seu cotidiano, refletido em situações negativas a respeito do uso prolongado do álcool.

Entende-se que diante desse grupo o conteúdo representacional acerca da consumação de álcool que se mostra mais destaque são as sensações negativas. A dependência do álcool coloca os idosos e sua família em um maior risco de vulnerabilidade para desenvolver problemas físicos, psicológicos e sociais, que nem sempre são detectados pelos profissionais nos diversos serviços de saúde, fazendo com que o idoso com problemas de alcoolismo não receba o tratamento adequado, comprometendo sua qualidade de vida.

Orientar as famílias dos alcoolistas também é uma necessidade, uma vez que todos os membros familiares podem adoecer com essas situações, sendo o apoio familiar de suma importância para a reestruturação do usuário de álcool.

Assim, diante do envelhecimento populacional, percebe-se a necessidade de desenvolver estratégias protetoras que possam oferecer um suporte à população idosa crescente. Por essa razão é importante compreender os impactos do uso abusivo do álcool pelo idoso, os problemas associados e suas representações a respeito do consumo de bebidas alcoólicas no intuito de promover uma melhor qualidade de vida.

\section{Referências}

1. Senger AEV, Ely LS, Gandolfi T, Schneider RH, Gomes I, Carli GA. Alcoolismo e tabagismo em idosos: relação com ingestão alimentar e aspectos socioeconômicos. Rev Bras Geriatr Gerontol. 2011;14(4):713-9.

2. Organização Mundial de Saúde (OMS). Resumo Relatório Mundial de Envelhecimento e Saúde [Internet]. [Acesso 31 ago 2016]. Disponível em:
http://sbgg.org.br/wp-content/uploads/2015/10/OMSENVELHECIMENTO-2015-port.pdf

3. Kano MY, Santos MA, Pillon SC. Uso do álcool em idosos: validação transcultural do Michigan Alcoholism Screening Test - Geriatric Version (MAST-G). Rev Esc Enferm USP. [Internet]. 2014;48(4):649-56.

4. Conselho Nacional Antidrogas (BR). A Política nacional sobre drogas. Brasília: Presidência da República; 2005.

5. Cardoso LGV, Melo APS, Cesar CC. Prevalência do consumo moderado e excessivo de álcool e fatores associados entre residentes de Comunidades Quilombolas de Vitória da Conquista, Bahia, Brasil. Ciênc Saúde Coletiva. 2015;20(3):809-20.

6. Ministério da Saúde (BR). Política Nacional de Saúde Integral da População Negra. Secretaria Especial de Políticas de Promoção da Igualdade Racial - SEPPIR, Brasília; 2007. 70 p.

7. Pianelli C, Abric JC, Saad F. Rôle des representations socials préexistantes dans les processus d'ancrageet structuration d'une nouvelle representation. CIPS 2010;86:241-74.

8. Moscovici S. A representação social da psicanálise. Rio de Janeiro: Zahar; 1978.

9. Abric JC. A abordagem estrutural das representações sociais. In: Moreira ASP, Oliveira DC, organizadores. Estudos interdisciplinares de representação social. 2.ed. Goiânia: AB; 2000. p. 27-38.

10. Abric JC. A abordagem estrutural das representações sociais: desenvolvimentos recentes. In: Campos PHF, Loureiro MCS, organizadores. Representações sociais e práticas educativas. Goiânia: Editora UCG; 2003. p. 37-57. 11. Abric JC. La recherché du noyau central et la zone muette des representations sociales. In: Abric JC, editor. Méthodes d'études des eepresentationssociales. Ramonville Saint-Agne; 2003. p. 60-80.

12. Instituto Brasileiro de Geografia e Estatística (IBGE). Sinopse do Censo Demográfico. 2010 [Acesso 28 ago 2016]. Disponível em: http://www.censo2010.ibge.gov.br/ sinopse/.

13. Instituto Brasileiro de Geografia e Estatística (IBGE). Síntese de Indicadores Sociais: uma análise das condições de vida da população brasileira. Rio de Janeiro: IBGE; 2012. [Acesso 28 ago 2016]. Disponível em: 48 ftp://ftp.ibge.gov.br/Indicadores_Sociais/ Sintese_de_Indicadores_Sociais_2012/SIS_2012.pdf 14. Meira SS, Vilela ABA, Casotti CA, Nascimento JC, Andrade $C B$. Idosos em estado de corresidência em um município do interior da Bahia. O Mundo Saúde. 2015;39(2):201-9.

15. Indicador de Analfabetismo Funcional (INAF). Analfabetismo, uma triste realidade. [Acesso 28 ago 2016]. Disponível em: http://www.ipm.org.br/ptbr/ programas/inaf/relatoriosinafbrasil/Paginas/default.aspx. 
16. World Health Organization (WHO). Status report on alcohol and health 2014. Geneva: World Health Organization; 2014.

17. Minayo MC, Deslandes SF. A complexidade das relações entre drogas, álcool e violência. Cad Saúde Pública. 1998;14(1):35-42. http://dx.doi.org/10.1590/ S0102-311X1998000100011.

18. Marques SC, Oliveira DCD, Gomes AMT. AIDS e representações sociais: uma análise comparativa entre subgrupos de trabalhadores. Psicologia: teoria e prática. 2004;6(spe): 91-104.

19. Flament C, Jodelet D. Estrutura e dinâmica das representações sociais. As representações sociais. 2001. 73-186.

20. Pedrosa AAS, Camacho LAB, Passos SRL, Oliveira RVC. Consumo de álcool entre estudantes universitários. Cad Saúde Pública. [Internet]. 2011 [Acesso 1 set 2016];27(8):1611-21. Disponível em: http://dx.doi.org/ 10.1590/S0102-311X1998000100011.

21. Souza J, Carvalho AMP. Repercussões do ambiente familiar alcoolista para o desenvolvimento da criança. Relato de caso. Pediatria Moderna. [Internet]. 2010 [Acesso 23 fev 2012]; 46(3): [cerca 5 p]. Disponível em: http://www.moreirajr.com.br/revistas.asp?id_materia $=4$ 357\&fase $=$ imprime.

22. Oliveira CD, Marques SC, Tosoli AM. Análise das evocações livres: uma técnica de análise estrutural das representações sociais. In: Moreira AP, Camargo BV, Jesuíno JC, Nóbrega SM, organizadores. Perspectivas teórico-metodológicas em representações sociais. João Pessoa: Ed. UFPB; 2005.
Recebido: 06.03.2017

Aceito: 15.01 .2019
Autor correspondente:

Thainara Araujo Franklin

E-mail: thainarafranklin@hotmail.com

(iD) https://orcid.org/0000-0003-2065-5090
Copyright $\odot 2019$ SMAD, Rev. Eletrônica Saúde Mental Álcool Drog. Este é um artigo de acesso aberto distribuído sob os termos da Licença Creative Commons CC BY-NC.

Esta licença permite que outros remixem, adaptem e criem a partir do seu trabalho para fins não comerciais, e embora os novos trabalhos tenham de lhe atribuir o devido crédito e não possam ser usados para fins comerciais, os usuários não têm de licenciar esses trabalhos derivados sob os mesmos termos. 